\title{
$\mathbb{N}<\mathrm{TT}$
}

Karlsruhe Institute of Technology

\section{Neutron Activation System for the European ITER Test Blanket Modules}

A. Klix¹, M. Angelone ${ }^{2}$, U. Fischer ${ }^{1}$, D. Gehre ${ }^{3}$, B. Ghidersa ${ }^{1}$, G. Kleizer ${ }^{4}$, P. Raj ${ }^{1}$, Th. Reimann¹, I. Rovni ${ }^{4}$, T. Rücker ${ }^{5}$, K. Tian ${ }^{1}$

${ }^{1}$ Karlsruhe Institute of Technology, Karlsruhe, Germany

${ }^{4}$ Budapest University of Technology and Economics, Budapest, Hungary

${ }^{2}$ ENEA Frascati, Frascati, Italien

${ }^{5}$ University of Applied Sciences Zittau-Görlitz, Zittau, Germany

${ }^{3}$ Technische Universität Dresden, Dresden, Germany

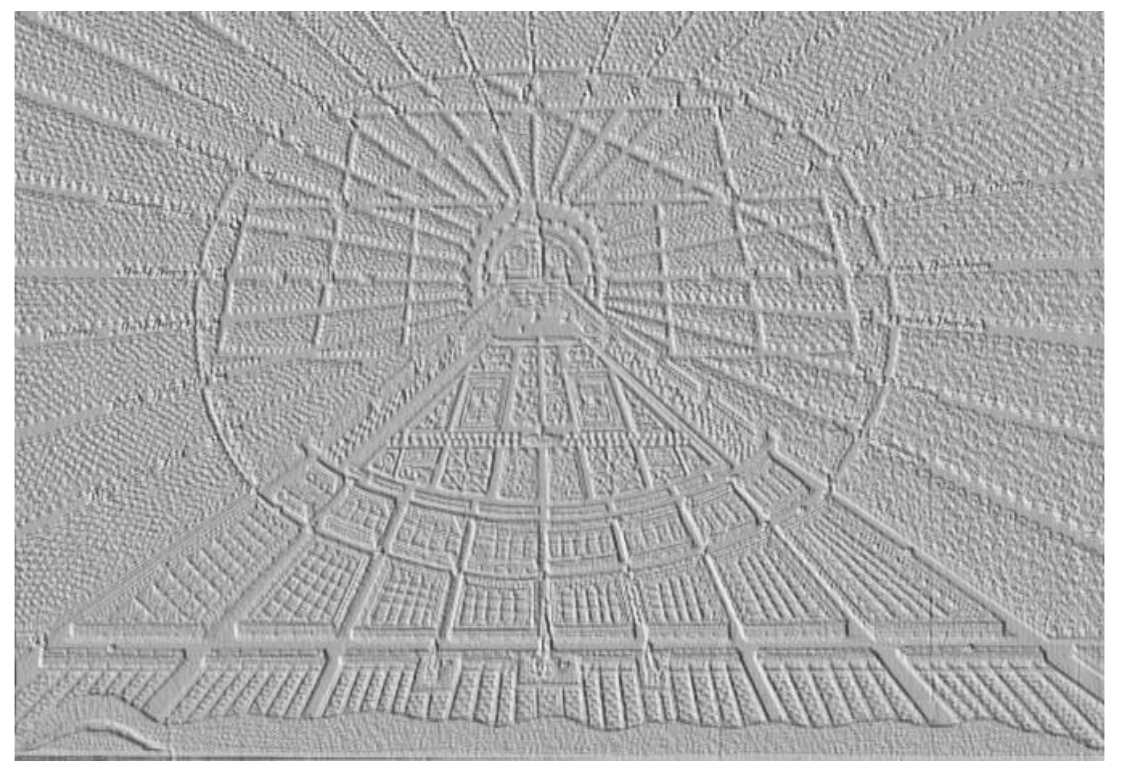


Breeding blanket concepts (EU) to be tested in ITER

\begin{tabular}{|l|l|l|}
\hline & $\begin{array}{l}\text { Helium-Cooled Lithium-Lead } \\
\text { (HCLL) }\end{array}$ & $\begin{array}{l}\text { Helium-Cooled Pebble Bed } \\
\text { (HCPB) }\end{array}$ \\
\hline Tritium breeder & Liquid lithium lead & $\mathrm{Li}_{4} \mathrm{SiO}_{4}$ or $\mathrm{Li}_{2} \mathrm{TiO}_{3}$ \\
\hline Neutron multiplier & Liquid lithium lead & Beryllium \\
\hline Coolant & Helium & Helium \\
\hline
\end{tabular}

Test Blanket Modules will be inserted in equatorial port \#16

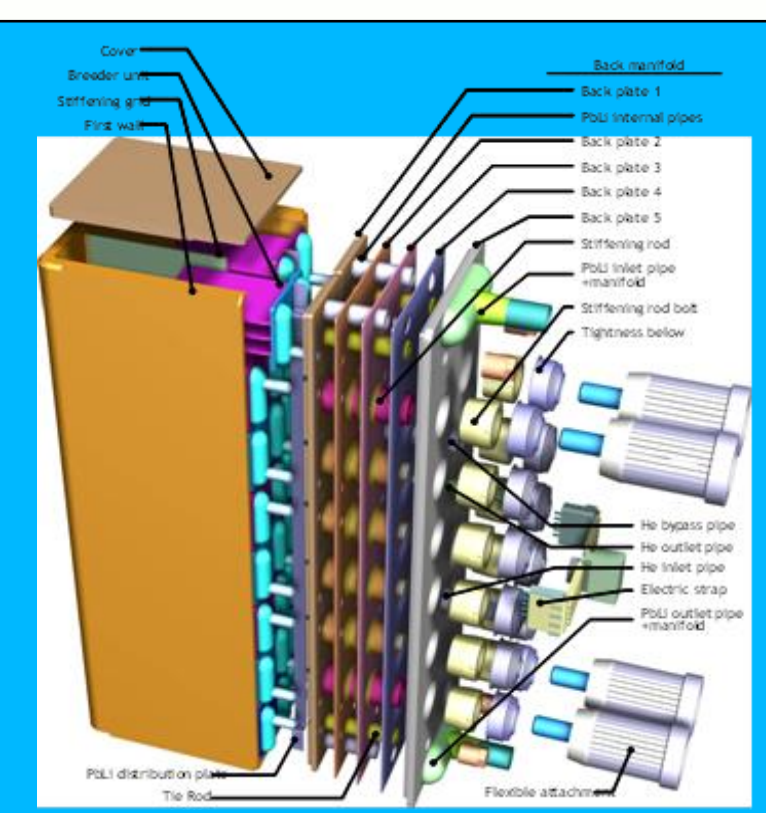

HCLL TBM

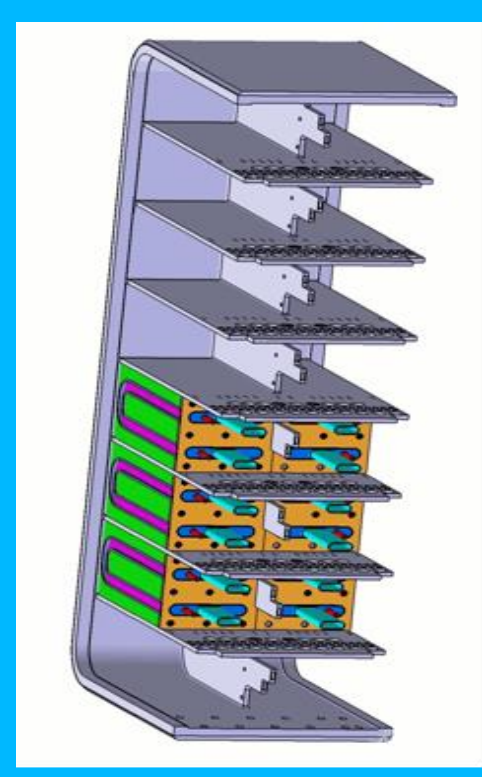

HCPB TBM

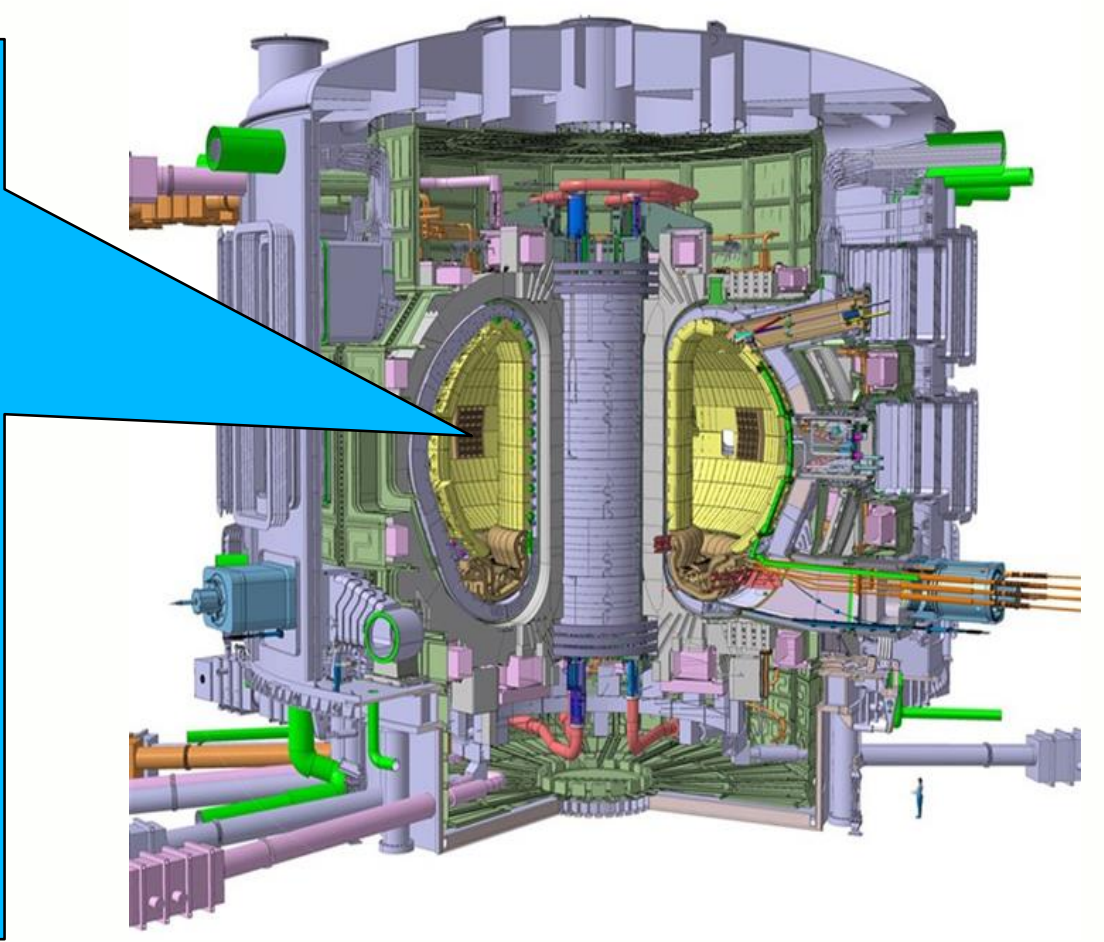




\section{Neutronics instrumentation for the ITER TBM Conditions in the TBM}

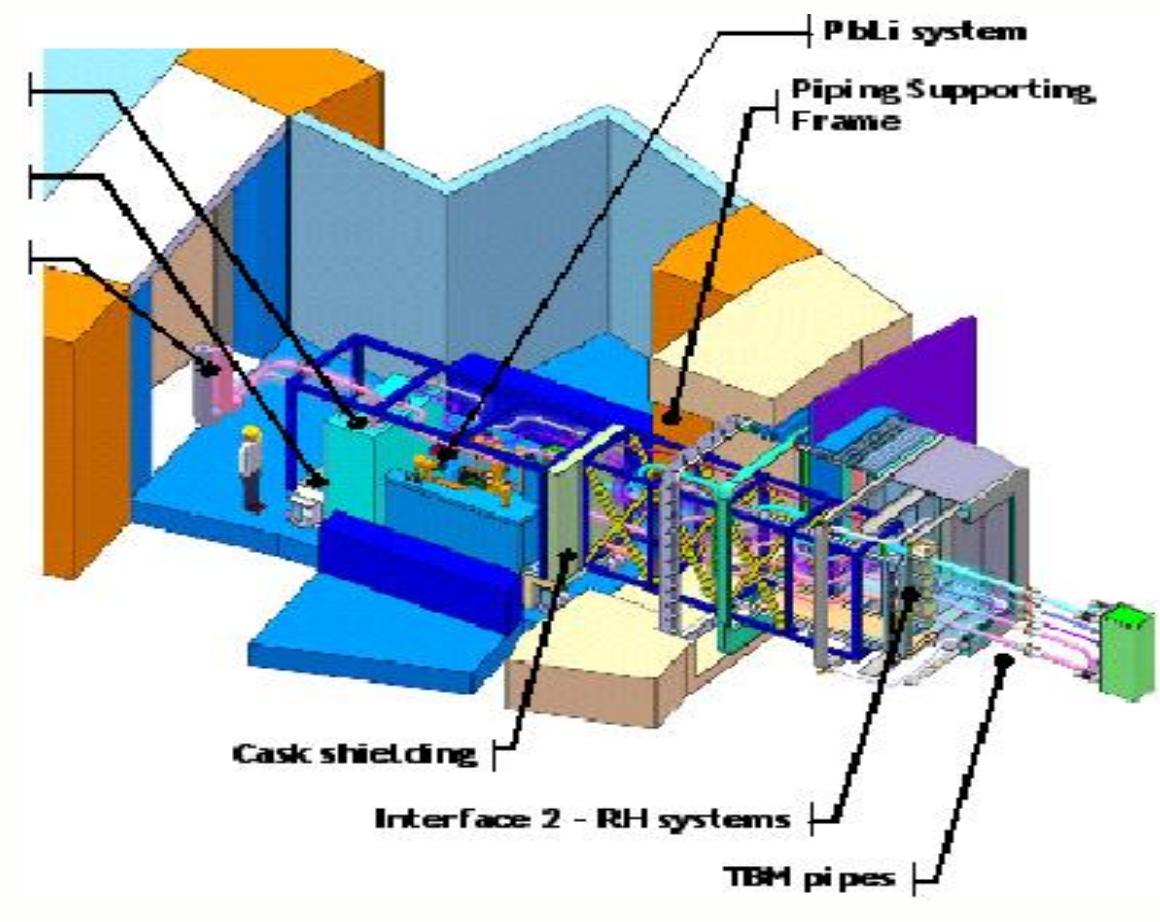

Possible candidates for neutron flux measurements:
Conditions in the TBM bad for any kind of detectors / diagnostics

- neutrons $10^{8} \sim 10^{14} \mathrm{~cm}^{-2} \mathrm{~s}^{-1}$

- from thermal to $15 \mathrm{MeV}$

- photons $10^{7} \sim 10^{13} \mathrm{~cm}^{-2} \mathrm{~s}^{-1}$

$-300 . .550^{\circ} \mathrm{C}$

- magnetic fields $\sim 4 \mathrm{~T}$

- difficult access

- little space
$>$ neutron activation system

$>$ miniature fission chambers

$>$ diamond detectors

> silicon carbide detectors

$>$ self-powered neutron detectors

$>$ miniature fission chambers 


\section{Neutron activation system}

\section{General considerations}

$>$ Neutron field and surface heat flux fundamental for establishing test conditions of the TBMs, will be measured by several ITER diagnostic systems

$>$ Breeder and multiplier modify the neutron field substantially inside the TBM

$>$ Measurement of the neutron field inside the TBM therefore essential for fulfillment of the scientific mission

\section{Particular importance for Tritium accountancy!}

\section{ITER TBM neutronics experiments will allow to check}

$>$ High-fidelity calculational tools

$>$ Modelling of heterogeneous complicated structures under fusion reactor relevant conditions 


\section{TBM Neutron Activation System}

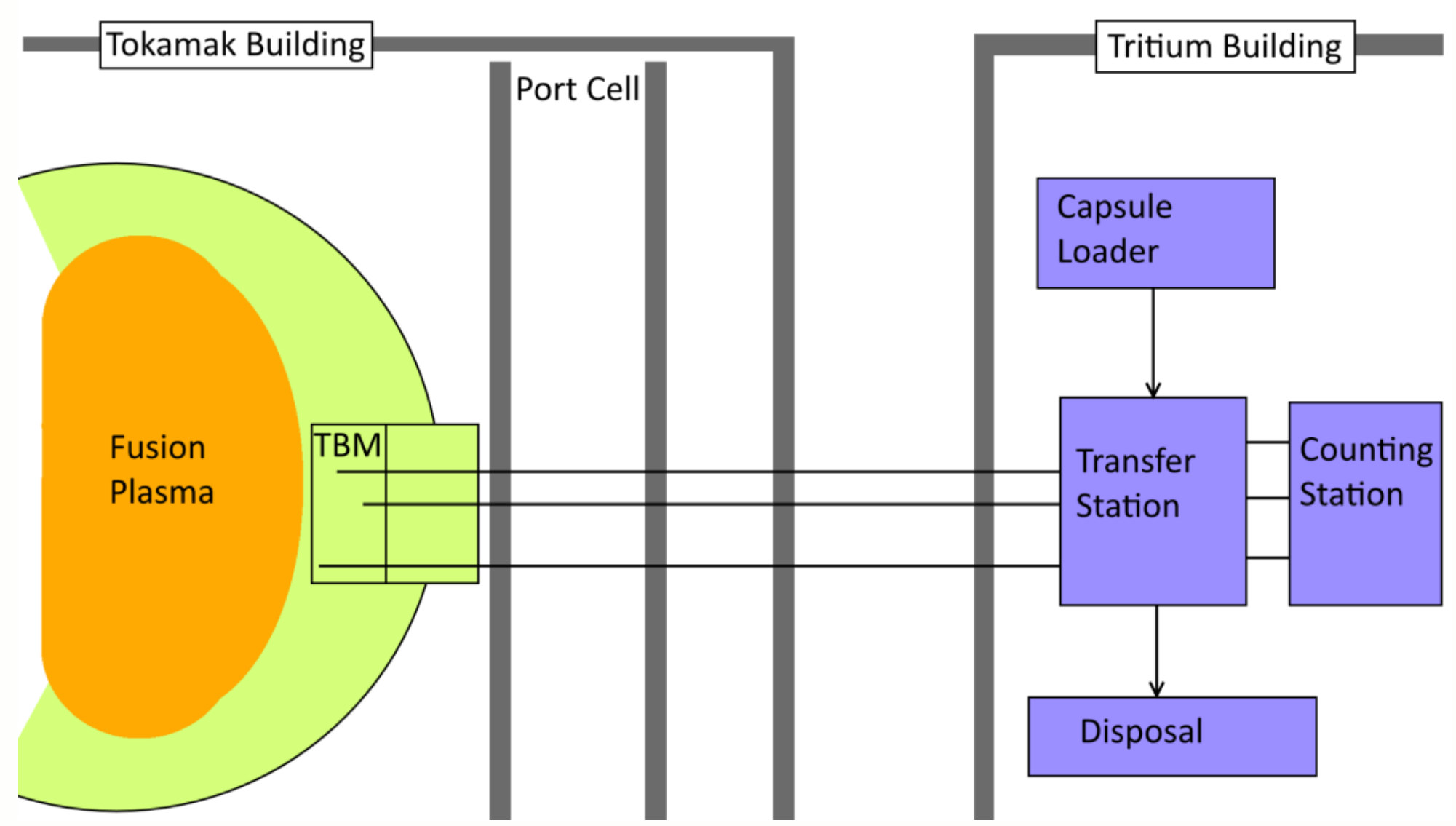

\section{Layout of a TBM neutron activation system}




\section{TBM Neutron Activation System}

\section{Mechanical part}

Mechanical test system under construction at INR

Preliminary engineering assessment completed

- TBM-NAS similar to ITER NAS due to nature of problem to be solved

- Must be driven by He; $\mathrm{N}_{2}$ etc. would be no option

- Expect three or four measurement positions in each TBM (HCLL and HCPB)

\section{Neutronics part}

- Selection of suitable activation materials

- Test measurements with neutron generator and pneumatic transport system

- Optimization of mass ratios and rabbit design

- Investigation of measurement uncertainties 


\section{TBM Neutron Activation System}

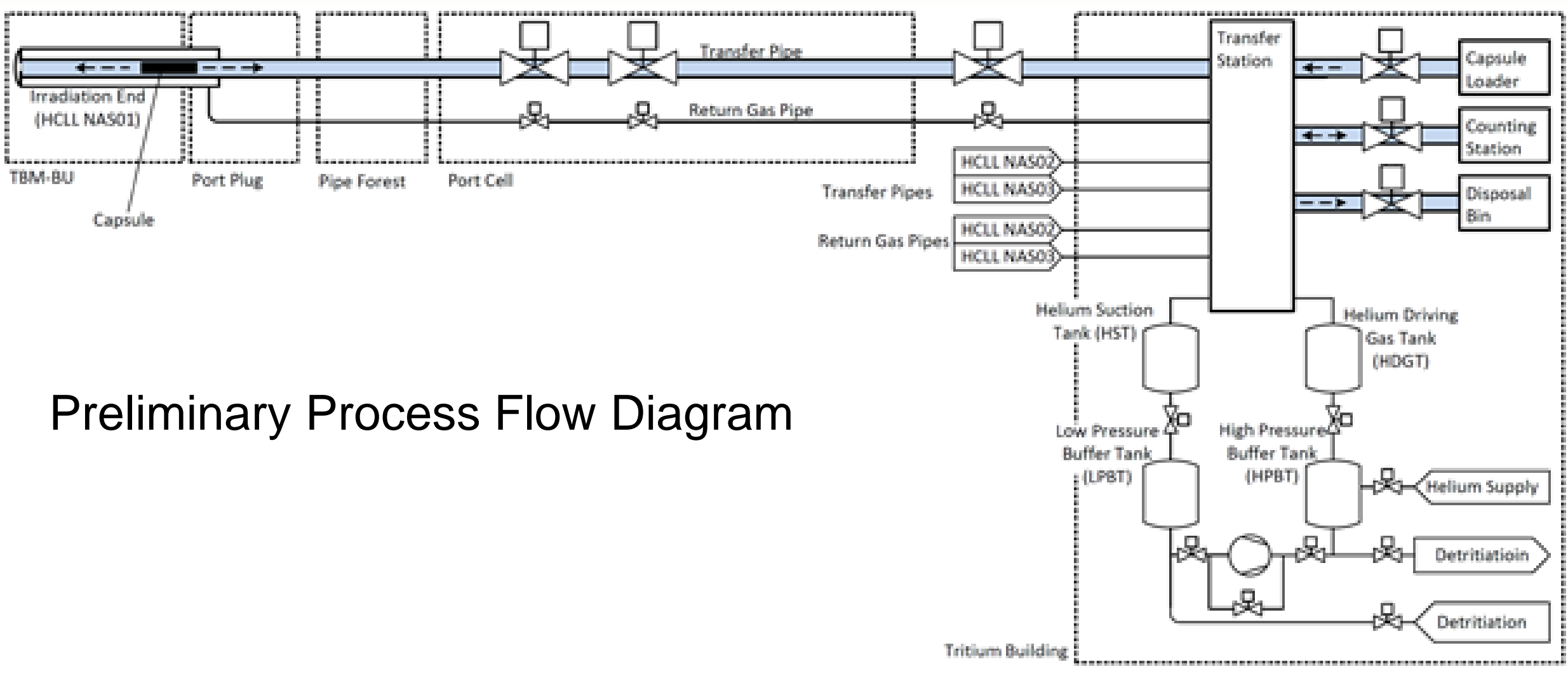

$>$ TBM-NAS similar to ITER NAS due to nature of problem to be solved

$>$ Must be driven by He; $\mathrm{N}_{2}$ etc. would be no option

$>$ Expect three or four measurement positions in each TBM (HCLL and HCPB) 


\section{TBM Neutron Activation System}

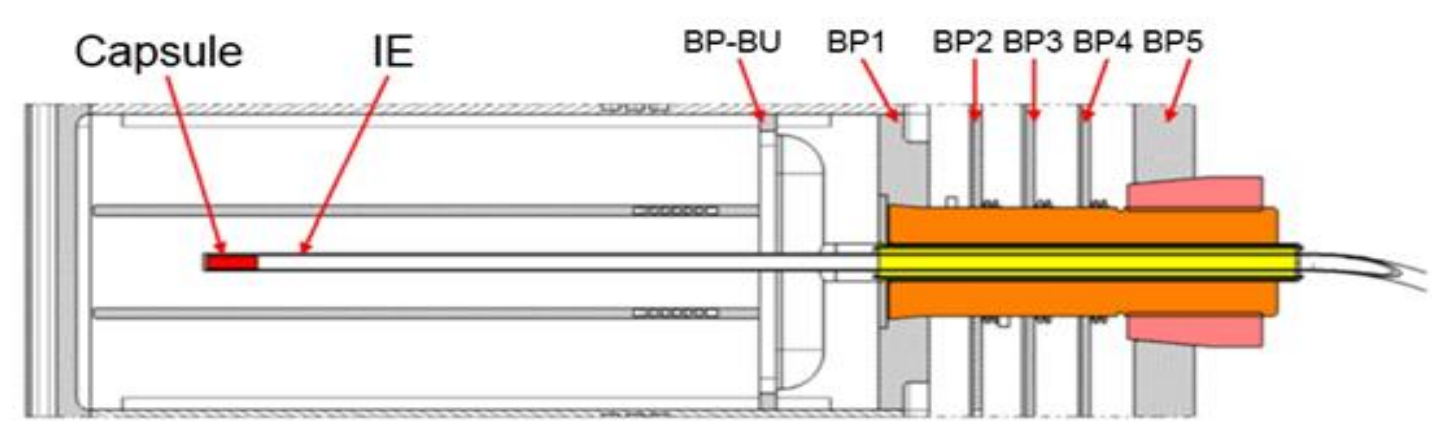

\section{Position of one IE in HCLL TBM}

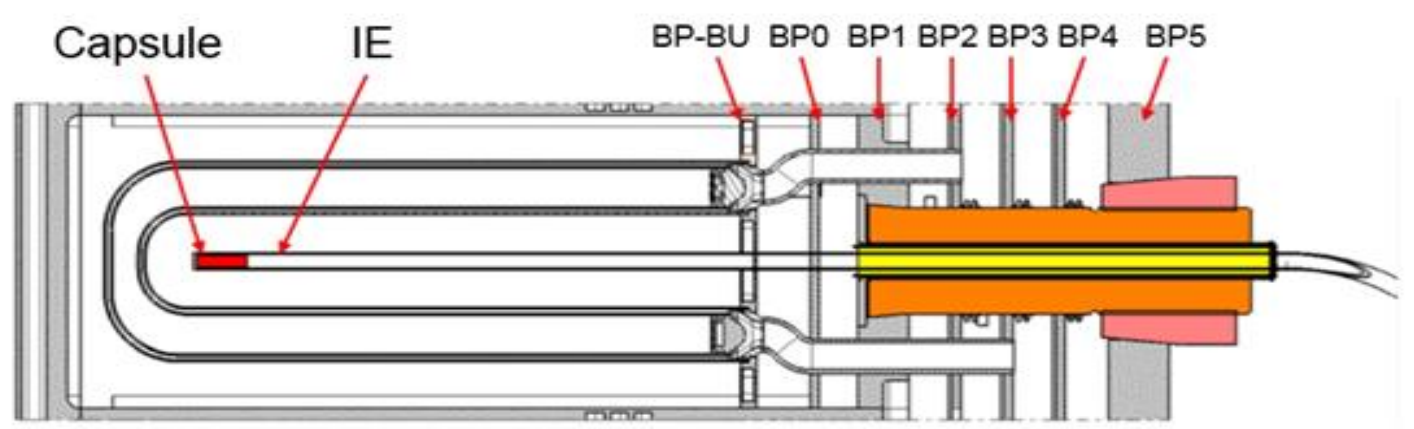

Position of one IE in HCPB TBM
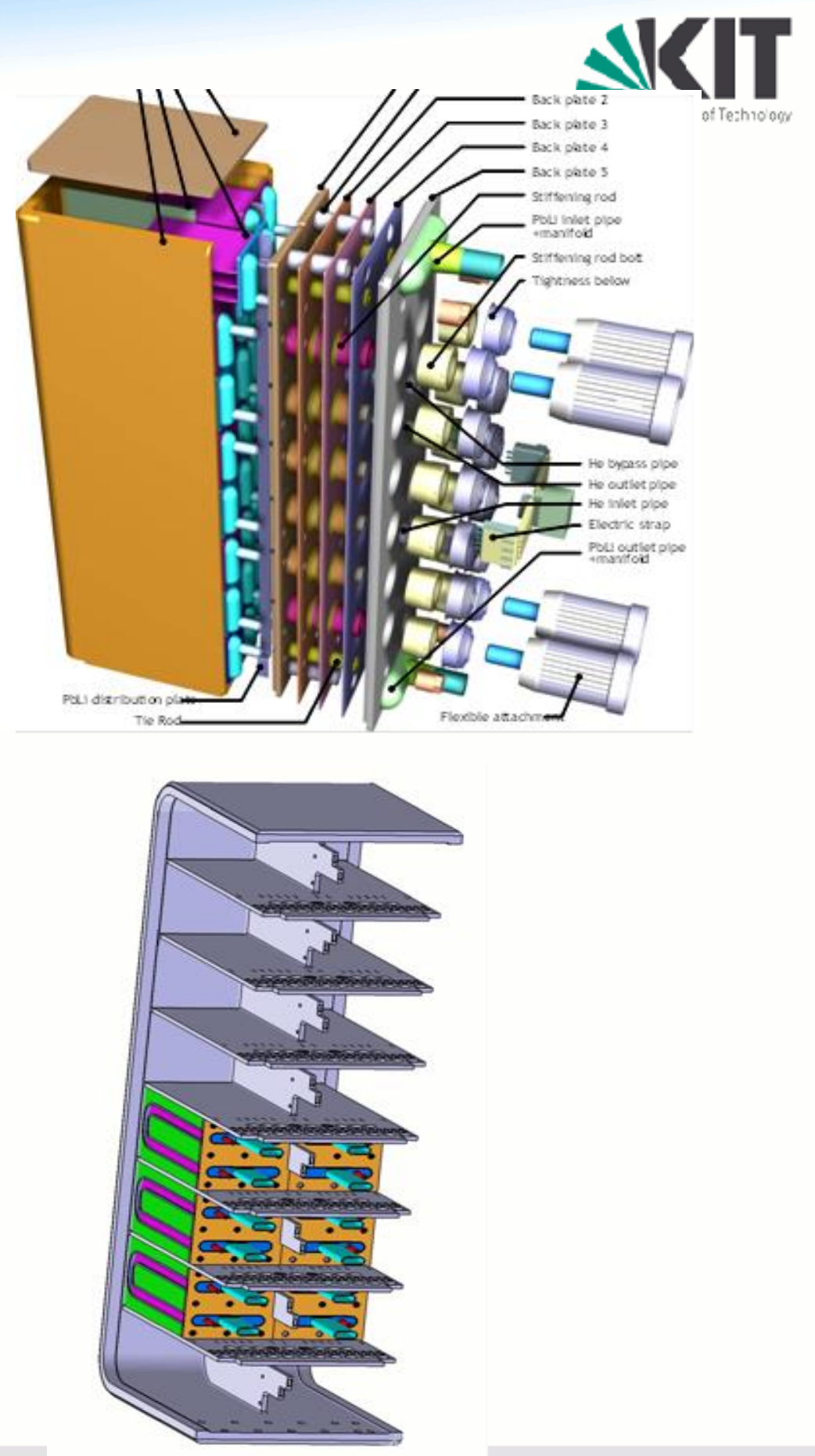

Institute of Neutron Physics
and Reactor Technology 


\section{Neutron activation test system at TUD-NG}

designed in collaboration with Technical University of Dresden

Utilizing intensive DT neutron generator

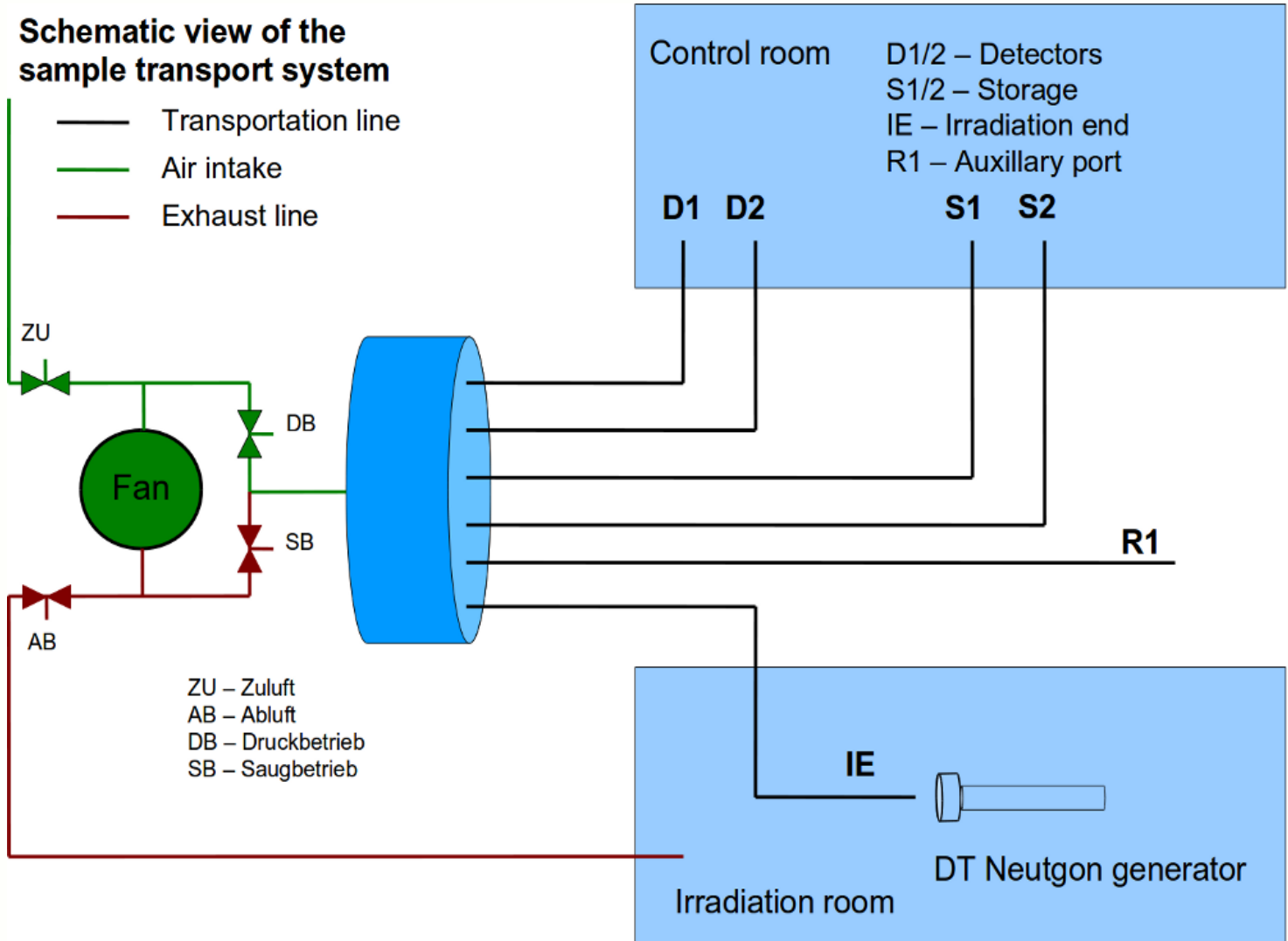

$>$ Testing of suitable measurement regimes

> Testing of suitable gamma ray detectors (HPGe, CZT,...)

$>$ Optimization of mass ratios and rabbit design

$>$ Investigation of measurement uncertainties

> Simultaneous gamma ray measurement of all materials in activation probe In TBM NAS: no manual processing of activation probe possible 


\section{Selected activation materials: $\mathrm{Ce}, \mathrm{Al}, \mathrm{Cr}, \mathrm{Nb},(\mathrm{Au})$}

\begin{tabular}{|c|c|c|c|}
\hline $\begin{array}{l}\text { Dosimetry } \\
\text { reaction }\end{array}$ & $\begin{array}{l}\text { Half-life } \\
\text { (sec) }\end{array}$ & $\begin{array}{l}\text { Approx. } \\
\text { threshold } \\
\text { energy (MeV) }\end{array}$ & Gamma-ray energy / Intensity of gamma line \\
\hline${ }^{140} \mathrm{Ce}(\mathrm{n}, 2 \mathrm{n}){ }^{139 \mathrm{~m}} \mathrm{Ce}$ & 56.1 & 10 & $754.2 / 0.9242$ \\
\hline${ }^{140} \mathrm{Ce}(\mathrm{n}, \mathrm{a}){ }^{137 \mathrm{~m} \mathrm{Ba}}$ & 153.12 & 12 & $661.7 / 0.9007$ \\
\hline${ }^{27} \mathrm{Al}(\mathrm{n}, \mathrm{g}){ }^{28} \mathrm{Al}$ & 134.46 & -- & $1778.7 / 1.00$ \\
\hline${ }^{27} \mathrm{Al}(\mathrm{n}, \mathrm{p}){ }^{27} \mathrm{Mg}$ & 567.48 & 4.5 & $843.7 / 0.718 \quad 1014.4 / 0.282$ \\
\hline${ }^{52} \mathrm{Cr}(\mathrm{n}, \mathrm{p}){ }^{52} \mathrm{~V}$ & 224.7 & 5.5 & $1434.1 / 1.000$ \\
\hline${ }^{53} \mathrm{Cr}(\mathrm{n}, \mathrm{p}){ }^{53} \mathrm{~V}$ & 97.2 & 6 & $1006.3 / 0.896 \quad 1289.5 / 0.1004$ \\
\hline${ }^{54} \mathrm{Cr}(\mathrm{n}, \mathrm{p}){ }^{54} \mathrm{~V}$ & 49.8 & 11 & $834.8 / 0.971 \quad 989.1 / 0.801 \quad 2259.3 / 0.456$ \\
\hline${ }^{54} \mathrm{Cr}(\mathrm{n}, \mathrm{p}){ }^{51} \mathrm{Ti}$ & 348.0 & 8.2 & $320.1 / 0.942$ \\
\hline${ }^{93} \mathrm{Nb}(\mathrm{n} . \mathrm{g}){ }^{94 \mathrm{~m} N b}$ & 375.6 & -- & $41.0 / 7.3 \mathrm{e}-4 \quad 871.1 / 4.95 \mathrm{e}-3$ \\
\hline${ }^{93} \mathrm{Nb}(\mathrm{n} . \mathrm{a}){ }^{90 \mathrm{~m} Y}$ & 11484 & 6.9 & $202.5 / 0.9725 \quad 479.5 / 0.9074$ \\
\hline${ }^{93} \mathrm{Nb}(\mathrm{n} . \mathrm{na}){ }^{89 \mathrm{~m} Y}$ & 15.663 & 12.5 & $909.0 / 0.9916$ \\
\hline${ }^{93} \mathrm{Nb}(\mathrm{n}, 2 \mathrm{n}){ }^{92 \mathrm{~m} N b}$ & 876960 & 9.5 & $934.5 / 0.9904$ \\
\hline
\end{tabular}

\begin{tabular}{|c|c|c|c|c|c|}
\hline \multicolumn{2}{|c|}{ Isotope } & \multirow{2}{*}{$\begin{array}{l}\text { Abundance } \\
(\%)\end{array}$} & \multirow{2}{*}{$\begin{array}{l}\begin{array}{l}\text { Melting } \\
\text { temp. }\left({ }^{\circ} \mathrm{C}\right)\end{array} \\
795\end{array}$} & \multicolumn{2}{|c|}{ Contributions to radio isotope } \\
\hline \multirow[t]{4}{*}{$\mathrm{Ce}$} & 136 & & & & \\
\hline & 138 & 0.25 & & & \\
\hline & 140 & 88.48 & & $\mathrm{n}, 2 \mathrm{n} \rightarrow{ }^{139 m} \mathrm{Ce} ; 99.99 \%$ & $\mathrm{n}, \mathrm{a} \rightarrow{ }^{137 m} \mathrm{Ba} ; 100 \%$ \\
\hline & 142 & 11.08 & & & \\
\hline Al & 27 & 100.0 & 660 & $\mathrm{n}, \mathrm{Y} \rightarrow{ }^{28} \mathrm{Al} ; 100 \%$ & $\mathrm{n}, \mathrm{p} \rightarrow{ }^{27} \mathrm{Mg} ; 100 \%$ \\
\hline \multirow[t]{4}{*}{$\mathrm{Cr}$} & 50 & 4.35 & 1907 & & \\
\hline & 52 & 83.79 & & $n, p \rightarrow{ }^{52} V ; 99.637 \%$ & \\
\hline & 53 & 9.50 & & $\mathrm{n}, \mathrm{p} \rightarrow{ }^{53} \mathrm{~V} ; 99.863 \%$ & \\
\hline & 54 & 2.36 & & $\mathrm{n}, \mathrm{p} \rightarrow{ }^{54} \mathrm{~V} ; 100 \%$ & $\mathrm{n}, \mathrm{a} \rightarrow{ }^{51} \mathrm{Ti} ; 100 \%$ \\
\hline $\mathrm{Nb}$ & 93 & 100.0 & 2468 & $\begin{array}{l}\mathrm{n}, \mathrm{Y} \rightarrow{ }^{94 \mathrm{~m} N b ; 100 \%} \\
\mathrm{n}, \mathrm{na} \rightarrow{ }^{89 m Y} ; 100 \%\end{array}$ & $\begin{array}{l}\mathrm{n}, \mathrm{a} \rightarrow{ }^{90 m Y} ; 100 \% \\
\mathrm{n}, 2 \mathrm{n} \rightarrow{ }^{92 \mathrm{~m} N b} ; 100 \%\end{array}$ \\
\hline
\end{tabular}
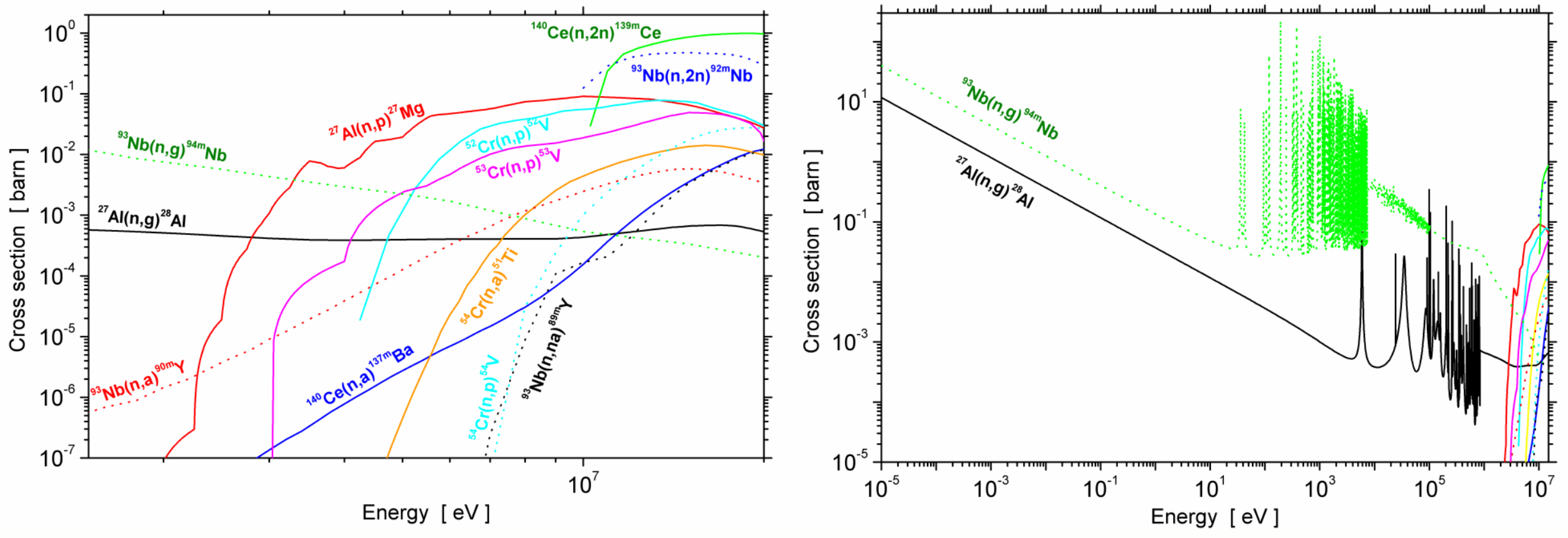


\section{Neutron Activation System test system at TUD-NG}
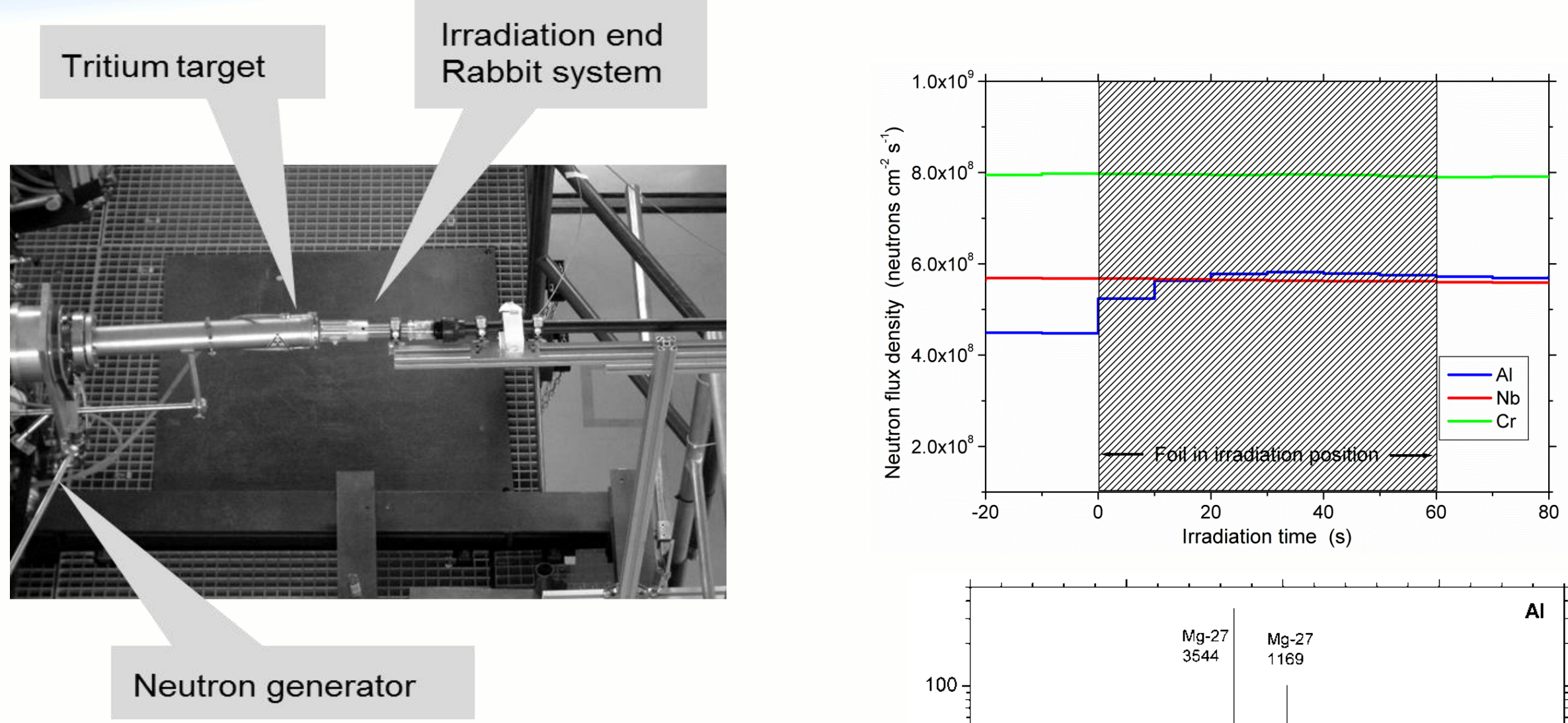

Test foils $10 \mathrm{~mm}$ diameter, $\sim 0.6 \mathrm{~g}$, material purity $>99.9 \%$

$>$ Irradiation time $60 \mathrm{~s}$, fluence at sample position $3.39-4.77 \times 10^{10} \mathrm{n} / \mathrm{cm}^{2}$

$>$ Transport time $16 . .23 \mathrm{~s}$

$>$ Gamma measurement $\mathrm{HPGe}, 30 \%$, ca. $5 \mathrm{~cm}$ distance 60 to $600 \mathrm{sec}$

Measurement uncertainty here: between 5 and $30 \%$

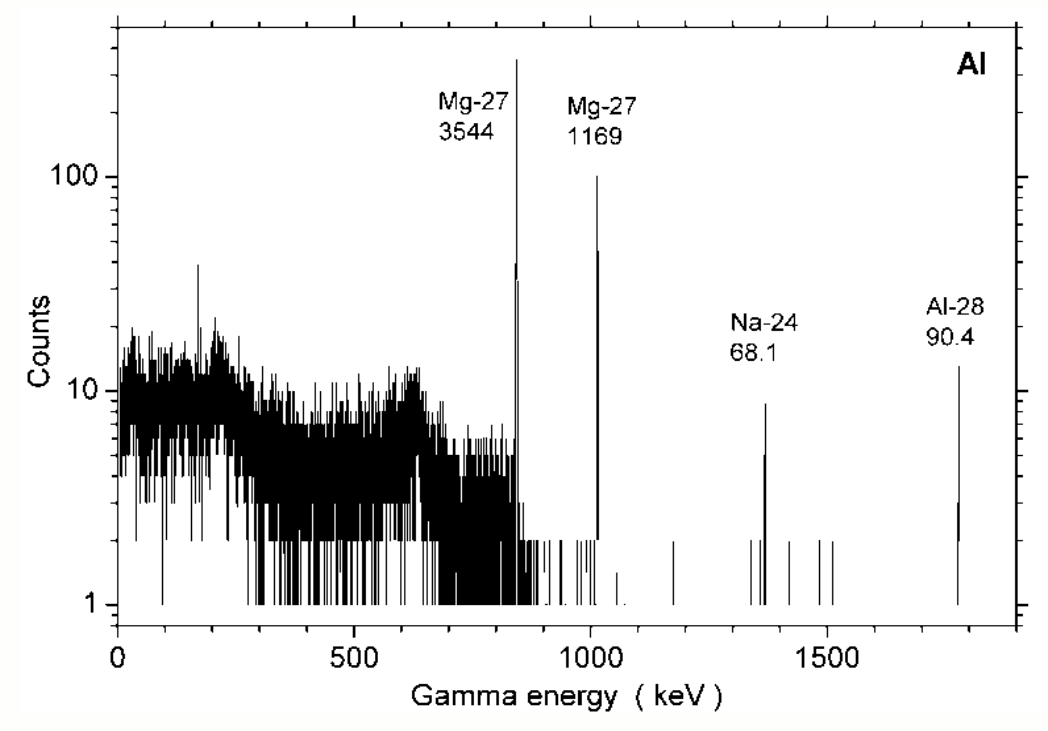




\section{Neutron Activation System test system at TUD-NG}

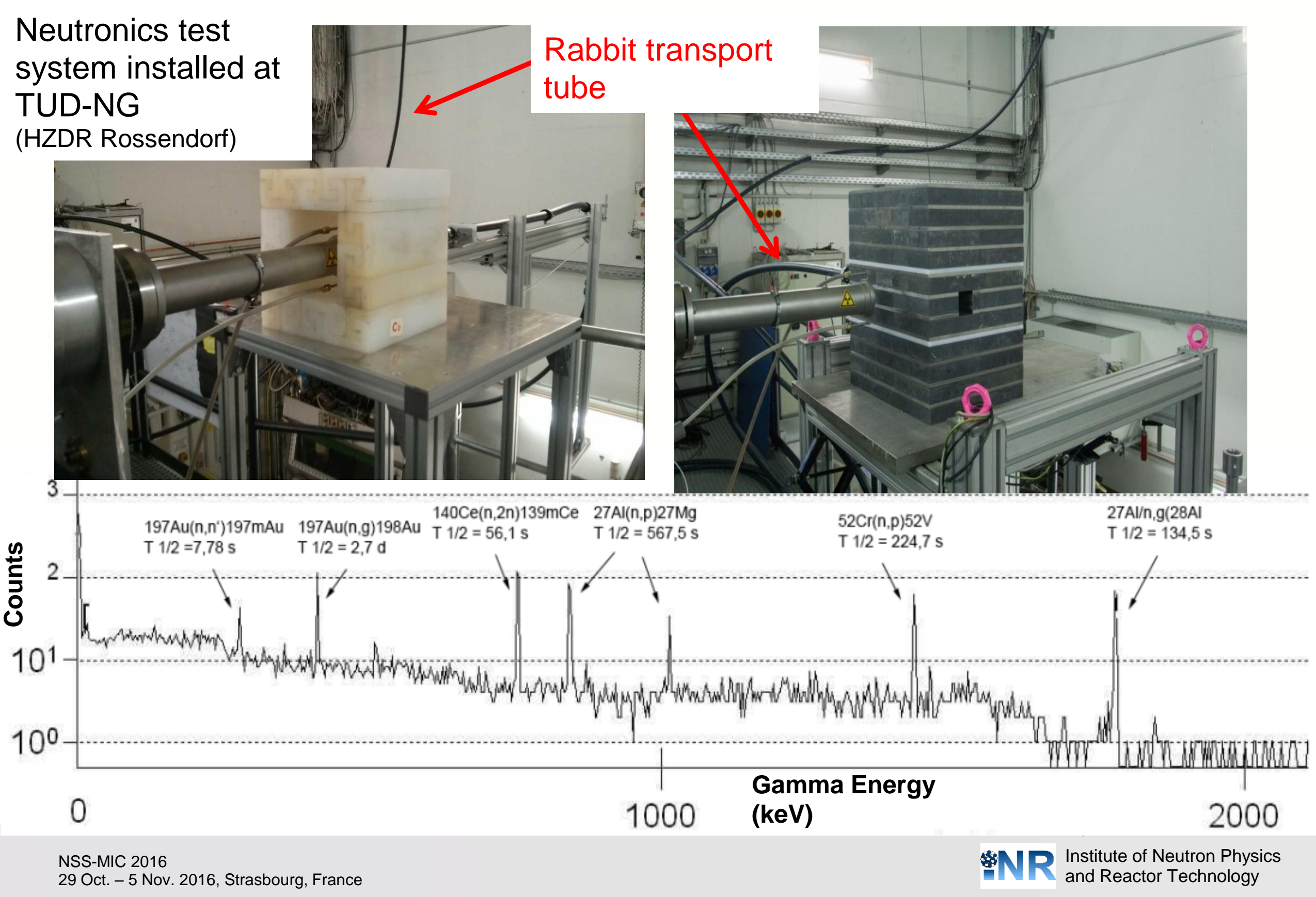




\section{Preliminary rabbit design}

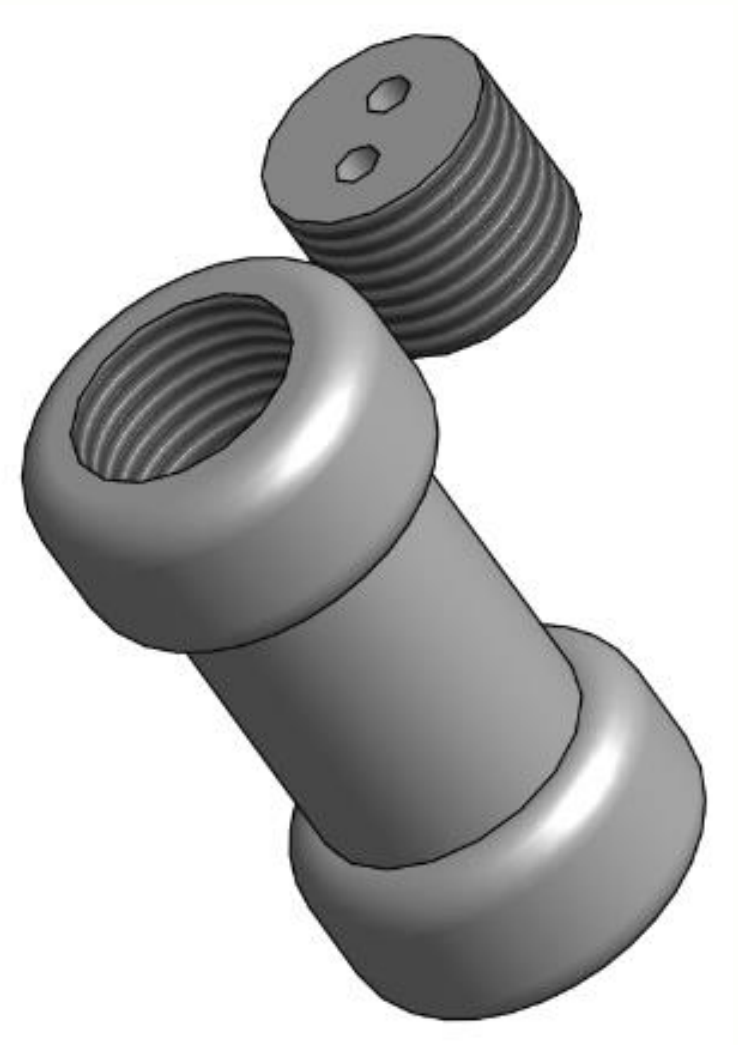

$>$ Dimensions: $8 \mathrm{~mm}$ diameter, $15 \mathrm{~mm}$ length

$>$ Bore for accommodation of activation materials $5 \mathrm{~mm}$ diameter

> Material: CFC or niobium

$>$ Niobium of interest since it can serve as activation material as well 


\section{Summary}

Neutron flux measurement inside the ITER TBM necessary for establishing test conditions of the TBM and the tritium breeding systems

Neutron activation system : - robust, can withstand harsh TBM conditions

- no calibration needed (except for gamma counting station)

- absolute values for local neutron field

- could be used in all ITER operation phases (DD+DT, low and high duty cycles)

- invasive and difficult to install

Preliminary engineering assessment done for the pneumatic transport system

Neutronics tests with activation probes done, further tests underway

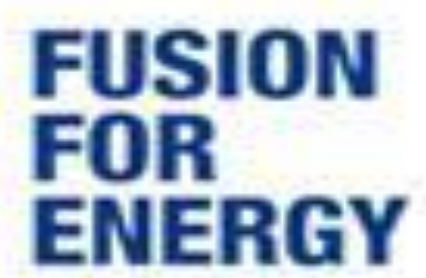

Disclaimer for parts of the work presented herein:

This work, supported by the European Communities under the Contract of Association between EURATOM and Forschungszentrum Karlsruhe, was carried out within the framework of the European Fusion Development Agreement. The views and opinions expressed herein do not necessarily reflect those of the European Commission. 